\title{
A NOVEL EDAS BASED METHOD FOR MULTIPLE ATTRIBUTE GROUP DECISION MAKING WITH PYTHAGOREAN 2-TUPLE LINGUISTIC INFORMATION
}

\author{
Tingting HE(D)1, Guiwu WEI ${ }^{1 *}$, Jianping $\mathrm{LU}^{1}$, \\ Jiang WUD2 ${ }^{2}$, Cun $\mathrm{WEI}^{2}$, Yanfeng $\mathrm{GUO}^{3}$ \\ ${ }^{1}$ School of Business, Sichuan Normal University, 610101 Chengdu, P.R. China \\ ${ }^{2}$ School of Statistics, Southwestern University of Finance and Economics, 611130 Chengdu, P.R. China \\ ${ }^{3}$ School of Finance, Southwestern University of Finance and Economics, 610074 Chengdu, P.R. China
}

Received 21 October 2019; accepted 19 April 2020

\begin{abstract}
In this article, we combine some fundamental theories of the Pythagorean 2-tuple linguistic sets (P2TLSs) with EDAS method and design the Pythagorean 2-tuple linguistic number (P2TLN) EDAS (P2TLN-EDAS) method for multiple attribute group decision making (MAGDM) issue. Firstly, the basic concepts of P2TLSs are introduced. Next, two aggregation operators of P2TLN are defined, and then the calculation steps of EDAS method are listed briefly. Furthermore, P2TLN-EDAS method is given for MAGDM problems and computing steps are proposed in detail. Finally, a computational example related to construction safety assessment is used to expound the effectiveness of the designed method. Meanwhile, we also carried out some comparative analysis between P2TLN-EDAS method and P2TLWA/P2TLWG operators and another P2TLN-TODIM method. The results show that P2TLN-EDAS method derives the same best alternative as P2TLWA, P2TLWG operators and P2TLN-TODIM method.
\end{abstract}

Keywords: MAGDM, P2TLSs, EDAS method, P2TLN-EDAS method, construction project.

JEL Classification: C43, C61, D81.

\section{Introduction}

The traditional EDAS method (Keshavarz Ghorabaee et al., 2015) has a wide range of application in dealing with MADM problems (Li \& Lu, 2019; Lu \& Wei, 2019; Morente-Molinera et al., 2018; Wang et al., 2019a) because it can solve conflicts between attributes. Then, Keshavarz Ghorabaee et al. (2016) found a way which uses the EDAS method to resolve these problems about the fuzzy MCDM problems for choosing supplier. Stevic et al. (2018) applied a newer approach of multi-criteria analysis with fuzzy EDAS method to pick out the most suitable manufacturer of PVC carpentry for the apartment refurbishing. Keshavarz Ghora-

${ }^{\star}$ Corresponding author. E-mail: weiguiwu1973@sicnu.edu.cn 
baee et al. (2017a) united the EDAS method with IT2FSs for evaluating supplier with respect to environmental criteria. Ilieva (2018) proposed two new algorithms of the EDAS method for MCGDM with fuzzy sets. Keshavarz Ghorabaee et al. (2017b) proposed a random EDAS method to solve the issue that the performance value of the alternative follows a normal distribution. Li et al. (2020) defined the EDAS method for MAGDM under q-rung orthopair fuzzy setting. Y. He et al. (2019) defined the EDAS method for MAGDM for green supplier selection with probabilistic uncertain linguistic sets.

In order to effectively to handle imprecise and ambiguous information (Wang, 2019; Wu et al., 2019a, 2019b) and some uncertain decision making issues (Lei et al., 2019; Wei et al., 2019), PFSs was presented by Yager (2014) at first. Peng and Yang (2015) devise the superiority \& inferiority sorting algorithm to deal with MAGDM issues in the context of PFSs. Ren, $\mathrm{Xu}$, and Gou (2016) stretched the TODIM to PFSs in order to address the MADM problems. In order to settle the issue of supplier selection, Chen (2018) defined a Novel PROMETHEE-based outranking approach for MADM with PFNs. Gao (2018) designed the Hamacher Prioritized operators for MADM using PFNs. Recently, G. W. Wei, Lu, Alsaadi, Hayat, and Alsaedi (2017) design P2TLSs for selecting enterprise resource planning system. Huang and Wei (2018) employed TODIM method to solve MADM under P2TLNs. T. T. He et al. (2019) developed the P2TLN-Taxonomy method to choose the medical instrument supplier.

However, it is obvious that there is no research on EDAS method of P2TLNs at present. Therefore, it is necessary to consider P2TLN-EDAS method. This study aims to propose a P2TLN-EDAS method to study MAGDM issues more effectively. The innovativeness of the paper can be summarized: (1) the P2TLN-EDAS method is proposed to solve the MAGDM issues under P2TLSs; (2) a case study for construction safety assessment is given to show the designed approach; and (3) some comparative studies are designed with some existing methods.

The rest part of this study is planned as below: In Section 1, the essential definitions of P2TLNs and two aggregation operators with P2TLNs are briefly described. P2TLN-EDAS approach is constructed and computational steps are listed in Section 2. A case study for construction safety assessment is given and some comparisons are also carried out to further demonstrate how effective and practical the proposed method is in Section 3. In last Section, we come to the conclusions of such work.

\section{Preliminaries}

\subsection{P2TLSs}

Wei et al. (2017) defined the P2TLSs on the foundation of the 2-tuple linguistic sets (Herrera \& Martinez, 2001) \& PFSs (Yager, 2014).

Definition 1 (Wei et al., 2017). In a P2TLS, $A$ in $X$ is defined

$$
P=\left\{\left(s_{p}(x), \phi\right),\left(u_{p}(x), v_{p}(x)\right), x \in X\right\},
$$

where $s_{p}(x) \in S, \phi \in[-0.5,0.5), u_{P}(x) \in[0,1]$ and $v_{P}(x) \in[0,1], u_{p}(x)$ and $v_{p}(x)$ meet the condition $0 \leq\left(u_{P}(x)\right)^{2}+\left(v_{P}(x)\right)^{2} \leq 1, \forall x \in X . u_{P}(x)$ is regarded as membership degree 
and $v_{P}(x)$ is regarded as non-membership degree of $x$ to 2-tuple linguistic information $\left(s_{p}(x), \phi\right) \cdot p=\left\langle\left(s_{p}, \phi\right),\left(u_{p}, v_{p}\right)\right\rangle$ is called a P2TLN.

Definition 2 (Wei et al., 2017). Assume that $p=\left\langle\left(s_{p}, \phi\right),\left(u_{p}, v_{p}\right)\right\rangle$ is a P2TLN, the scoring function of $p$ could be defined:

$$
\begin{aligned}
& S F(p)=\Delta\left(\Delta^{-1}\left(s_{\alpha(p)}, \phi\right) \frac{1+\left(u_{p}\right)^{2}-\left(v_{p}\right)^{2}}{2}\right), \\
& \Delta^{-1}(S F(p)) \in[1, t] .
\end{aligned}
$$

Definition 3 (Wei et al., 2017). Assume that $p=\left\langle\left(s_{p}, \phi\right),\left(u_{p}, v_{p}\right)\right\rangle$ is a P2TLN, the accuracy function of $p$ could be defined:

$$
\begin{aligned}
& A F(p)=\Delta\left(\Delta^{-1}\left(s_{\alpha(p)}, \phi\right) \frac{\left(u_{p}\right)^{2}+\left(v_{p}\right)^{2}}{2}\right), \\
& \Delta^{-1}(A F(p)) \in[1, t] .
\end{aligned}
$$

Definition 4 (Wei et al., 2017). Assume that $p_{1}=\left\langle\left(s_{p_{1}}, \phi_{1}\right),\left(u_{p_{1}}, v_{p_{1}}\right)\right\rangle$ and $p_{2}=\left\langle\left(s_{p_{2}}, \phi_{2}\right)\right.$, $\left.\left(u_{p_{2}}, v_{p_{2}}\right)\right\rangle$ are two P2TLNs, $S F\left(p_{1}\right)=\Delta\left(\Delta^{-1}\left(s_{\alpha\left(p_{1}\right)}, \phi_{1}\right) \cdot \frac{1+\left(u_{p_{1}}\right)^{2}-\left(v_{p_{1}}\right)^{2}}{2}\right), S F\left(p_{2}\right)=$ $\Delta\left(\Delta^{-1}\left(s_{\alpha\left(p_{2}\right)}, \phi_{2}\right) \cdot \frac{1+\left(u_{p_{2}}\right)^{2}-\left(v_{p_{2}}\right)^{2}}{2}\right)$ are scores functions of $p_{1}$ and $p_{2}$, respectively, and $A F\left(p_{1}\right)=\Delta\left(\Delta^{-1}\left(s_{\alpha\left(p_{1}\right)}, \phi_{1}\right) \cdot \frac{\left(u_{p_{1}}\right)^{2}+\left(v_{p_{1}}\right)^{2}}{2}\right), \quad A F\left(p_{2}\right)=\Delta\left(\Delta^{-1}\left(s_{\alpha\left(p_{2}\right)}, \phi_{2}\right)\right.$. $\left.\frac{\left(u_{p_{2}}\right)^{2}+\left(v_{p_{2}}\right)^{2}}{2}\right)$ are the accuracy function of $p_{1}$ and $p_{2}$, then

(1) if $S F\left(p_{1}\right)<S F\left(p_{2}\right), p_{1}<p_{2}$;

(2) if $S F\left(p_{1}\right)=S F\left(p_{2}\right), A F\left(p_{1}\right)<A F\left(p_{2}\right)$, then $p_{1}<p_{2}$;

(3) if $S F\left(p_{1}\right)=S F\left(p_{2}\right), A F\left(p_{1}\right)=A F\left(p_{2}\right)$, then $p_{1}=p_{2}$.

Definition 5 (Huang \& Wei, 2018). Let $p_{1}=\left\langle\left(s_{p_{1}}, \rho_{1}\right),\left(u_{p_{1}}, v_{p_{1}}\right)\right\rangle$ and $p_{2}=\left\langle\left(s_{p_{2}}, \rho_{2}\right),\left(u_{p_{2}}, v_{p_{2}}\right)\right\rangle$ be two P2TLNs, the normalized Hamming distance between $p_{1}$ and $p_{2}$ can be calculated:

$$
d\left(p_{1}, p_{2}\right)=\frac{1}{2 T}\left[\left(\mid \begin{array}{l}
\left(1+\left(u_{p_{1}}\right)^{2}-\left(v_{p_{1}}\right)^{2}\right) \cdot \Delta^{-1}\left(s_{p_{1}}, \rho_{1}\right)- \\
\left(1+\left(u_{p_{2}}\right)^{2}-\left(v_{p_{2}}\right)^{2}\right) \cdot \Delta^{-1}\left(s_{p_{2}}, \rho_{2}\right)
\end{array}\right)\right] .
$$




\subsection{The P2TLWA and P2TLWG operators}

In this part, two fusing operators with P2TLNs are introduced (Wei et al., 2017).

Definition 6 (Wei et al., 2017). Assume that $p=\left\langle\left(s_{p_{j}}, \phi_{j}\right),\left(u_{p_{j}}, v_{p_{j}}\right)\right\rangle(j=1,2, \ldots, n)$ is a set of P2TLNs. Thus, the P2TLWA operator could be defined:

$$
\begin{aligned}
& \text { P2TLWA }_{\omega}\left(p_{1}, p_{2}, \ldots, p_{n}\right)=\bigoplus_{j=1}^{n}\left(\omega_{j} p_{j}\right)= \\
& \left\langle\Delta\left(\sum_{j=1}^{n} \omega_{j} \Delta^{-1}\left(s_{p_{j}}, \phi_{j}\right)\right),\left(\sqrt{\left.\left.1-\prod_{j=1}^{n}\left(1-\left(u_{p_{j}}\right)^{2}\right)^{\omega_{j}}, \prod_{j=1}^{n}\left(v_{p_{j}}\right)^{\omega_{j}}\right)\right\rangle ;}\right.\right. \\
& \text { P2TLWG }\left(p_{1}, p_{2}, \ldots, p_{n}\right)=\bigotimes_{j=1}^{n}\left(\omega_{j} p_{j}\right)= \\
& \left\langle\Delta\left(\prod_{j=1}^{n} \Delta^{-1}\left(s_{p_{j}}, \phi_{j}\right)^{\omega_{j}}\right),\left(\prod_{j=1}^{n}\left(u_{p_{j}}\right)^{\omega_{j}} \sqrt{\left.\left.1-\prod_{j=1}^{n}\left(1-\left(v_{p_{j}}\right)^{2}\right)^{\omega_{j}}\right)\right\rangle}\right)\right.
\end{aligned}
$$

where $\omega=\left(\omega_{1}, \omega_{2}, \ldots, \omega_{n}\right)^{T}$ is the weight of $p_{j}(j=1,2, \ldots, n)$ and $\omega_{j}>0, \sum_{j=1}^{n} \omega_{j}=1$.

Example 1. There are four P2TLNs, such as $p_{1}=\left\langle\left(s_{2}, 0\right),(0.8,0.3)\right\rangle, p_{2}=\left\langle\left(s_{3}, 0\right),(0.5,0.6)\right\rangle$, $p_{3}=\left\langle\left(s_{1}, 0\right),(0.6,0.4)\right\rangle$ and $p_{4}=\left\langle\left(s_{5}, 0\right),(0.7,0.5)\right\rangle$. Supposing that their weight are $\omega=$ $\left(\omega_{1}, \omega_{2}, \omega_{3}, \omega_{4}\right)^{T}=(0.23,0.3,0.21,0.26)$, then according to (5) and (6), we have:

$$
\begin{aligned}
& \text { P2TLWA }_{\omega}\left(p_{1}, p_{2}, p_{3}, p_{4}\right)=\omega_{1} p_{1} \oplus \omega_{2} p_{2} \oplus \omega_{3} p_{3} \oplus \omega_{4} p_{4}= \\
& \left\langle\begin{array}{l}
\Delta(0.23 \times 2+0.3 \times 3+0.21 \times 1+0.26 \times 5), \\
\left.\left(\begin{array}{l}
1-\left(1-0.8^{2}\right)^{0.23} \times\left(1-0.5^{2}\right)^{0.3} \times\left(1-0.6^{2}\right)^{0.21} \times\left(1-0.7^{2}\right)^{0.26} \\
\left(0.3^{0.23} \times 0.6^{0.3} \times 0.4^{0.21} \times 0.5^{0.26}\right.
\end{array}\right)\right\rangle=
\end{array}\right. \\
& \left\langle\left(s_{3},-0.13\right),(0.6676,0.4481)\right\rangle ; \\
& \text { P2TLWG }\left(p_{1}, p_{2}, p_{3}, p_{4}\right)=\left(p_{1}\right)^{\omega_{1}} \otimes\left(p_{2}\right)^{\omega_{2}} \otimes\left(p_{3}\right)^{\omega_{3}} \otimes\left(p_{4}\right)^{\omega_{4}}= \\
& \left\langle\begin{array}{l}
\Delta\left(2^{0.23} \times 3^{0.3} \times 1^{0.21} \times 5^{0.26}\right), \\
\left.\left(\begin{array}{l}
0.8^{0.23} \times 0.5^{0.3} \times 0.6^{0.21} \times 0.7^{0.26}, \\
\left(\sqrt{1-\left(1-0.3^{2}\right)^{0.23} \times\left(1-0.6^{2}\right)^{0.3} \times\left(1-0.4^{2}\right)^{0.21} \times\left(1-0.5^{2}\right)^{0.26}},\right.
\end{array}\right)\right\rangle=
\end{array}\right. \\
& \left\langle\left(s_{2}, 0.48\right),(0.6317,0.4841)\right\rangle .
\end{aligned}
$$




\section{EDAS method for MAGDM with P2TLNs}

In such section, we lead into the P2TLN-EDAS method for handling the MAGDM problems. Suppose there are $m$ alternatives $\left\{P_{1}, P_{2}, \ldots P_{m}\right\}, n$ attributes $\left\{\alpha_{1}, \alpha_{2}, \ldots \alpha_{n}\right\}$ and $k$ experts $\left\{D_{1}, D_{2}, \ldots D_{k}\right\},\left\{\zeta_{1}, \zeta_{2}, \ldots \zeta_{k}\right\}$ is the expert's weight vector and $\left\{\omega_{1}, \omega_{2}, \ldots \omega_{n}\right\}$ is the attribute's weight which satisfy $\zeta_{t} \in[0,1], \omega_{j} \in[0,1], \sum_{t=1}^{k} \zeta_{t}=1, \sum_{j=1}^{n} \omega_{j}=1$. The computational procedure of the expanding method can be listed:

Step 1. Build the group decision matrix by some experts and finally form the decision matrix $G^{(k)}=\left(g_{i j}^{k}\right)_{m \times n}$ of each decision maker with P2TLNs.

$$
G^{(k)}=\left[g_{i j}^{k}\right]_{m \times n}=\left[\begin{array}{cccc}
g_{11}^{k} & g_{12}^{k} & \ldots & g_{1 n}^{k} \\
g_{21}^{k} & g_{22}^{k} & \ldots & g_{2 n}^{k} \\
\vdots & \vdots & \vdots & \vdots \\
g_{m 1}^{k} & g_{m 2}^{k} & \ldots & g_{m n}^{k}
\end{array}\right] .
$$

Step 2. Use P2TLWA or P2TLWG operator to fuse these group matrix and obtain the overall matrix $G=\left(g_{i j}\right)_{m \times n}$.

$$
\begin{aligned}
& G=\left[g_{i j}\right]_{m \times n}=\left[\begin{array}{cccc}
g_{11} & g_{12} & \ldots & g_{1 n} \\
g_{21} & g_{22} & \ldots & g_{2 n} \\
\vdots & \vdots & \vdots & \vdots \\
g_{m 1} & g_{m 2} & \ldots & g_{m n}
\end{array}\right]
\end{aligned}
$$

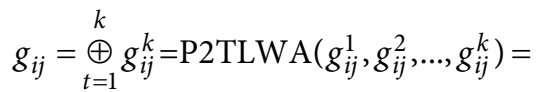

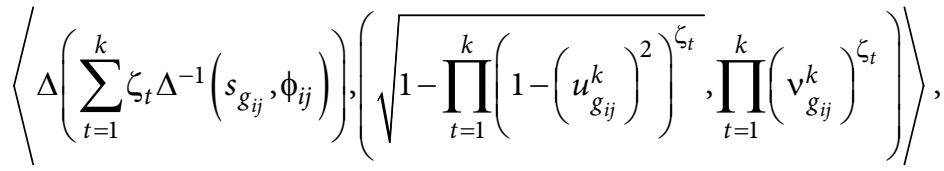

or

$$
\begin{aligned}
& g_{i j}=\bigotimes_{t=1}^{k} g_{i j}^{k}=\operatorname{P} 2 \operatorname{TLWG}\left(g_{i j}^{1}, g_{i j}^{2}, \ldots, g_{i j}^{k}\right)= \\
& \left\langle\Delta\left(\prod_{t=1}^{k} \Delta^{-1}\left(s_{g_{i j}}, \phi_{i j}\right)^{\zeta_{t}}\right),\left(\prod_{t=1}^{k}\left(u_{g_{i j}}^{k}\right)^{\zeta_{t}} \sqrt{1-\prod_{t=1}^{k}\left(1-\left(v_{g_{i j}}^{k}\right)^{2}\right)^{\zeta_{t}}}\right)\right\rangle .
\end{aligned}
$$

Step 3. Obtain AV based on all presented attributes:

According to Definition 6,

$$
A V=\left[A V_{j}\right]_{1 \times n}=\left[\frac{\sum_{i=1}^{m} g_{i j}}{m}\right]_{1 \times n}
$$

$$
\sum_{i=1}^{m} g_{i j}=\left\langle\Delta\left(\sum_{i=1}^{m} \Delta^{-1}\left(s_{g_{i j}}, \phi_{i j}\right)\right),\left(\sqrt{1-\prod_{i=1}^{m}\left(1-\left(u_{g_{i j}}\right)^{2}\right)}, \prod_{i=1}^{m}\left(v_{g_{i j}}\right)\right)\right\rangle .
$$


Thus

$$
\begin{aligned}
& A V=\left[A V_{j}\right]_{1 \times n}=\left[\frac{\sum_{i=1}^{m} g_{i j}}{m}\right]_{1 \times n}= \\
& \left\langle\Delta\left(\sum_{i=1}^{m} \frac{1}{m} \Delta^{-1}\left(s_{g_{i j}}, \phi_{i j}\right)\right),\left(\sqrt{\left.\left.1-\prod_{i=1}^{m}\left(1-\left(u_{g_{i j}}\right)^{2}\right)^{\frac{1}{m}}, \prod_{i=1}^{m}\left(v_{g_{i j}}\right)^{\frac{1}{m}}\right)\right\rangle} .\right.\right.
\end{aligned}
$$

Step 4. According to AV, PDA and NDA can be computed:

$$
\begin{gathered}
P D A_{i j}=\left[P D A_{i j}\right]_{m \times n}=\frac{\max \left(0,\left(g_{i j}-A V_{j}\right)\right)}{A V_{j}} ; \\
N D A_{i j}=\left[N D A_{i j}\right]_{m \times n}=\frac{\max \left(0,\left(A V_{j}-g_{i j}\right)\right)}{A V_{j}} .
\end{gathered}
$$

For the convenience of calculation, the formula in Definition 2 is utilized to calculate PDA and NDA values as follows.

$$
\begin{aligned}
& P D A_{i j}=\left[P D A_{i j}\right]_{m \times n}=\frac{\max \left(0,\left(s\left(g_{i j}\right)-s\left(A V_{j}\right)\right)\right)}{s\left(A V_{j}\right)} ; \\
& N D A_{i j}=\left[N D A_{i j}\right]_{m \times n}=\frac{\max \left(0,\left(s\left(A V_{j}\right)-s\left(g_{i j}\right)\right)\right)}{s\left(A V_{j}\right)} .
\end{aligned}
$$

Step 5. Acquire $S P_{i}$ and $S N_{i}$ :

$$
S P_{i}=\sum_{j=1}^{n} \omega_{j} P D A_{i j}, N P_{i}=\sum_{j=1}^{n} \omega_{j} N D A_{i j} .
$$

Step 6. Normalize the calculating results of Step5:

$$
N S P_{i}=\frac{S P_{i}}{\max \left(S P_{i}\right)}, N S N_{i}=1-\frac{S N_{i}}{\max \left(S N_{i}\right)} .
$$

Step 7. According to the results of $N S P_{i}$ and $N S N_{i}$, calculate the values of appraisal score (AS):

$$
A S_{i}=\frac{1}{2}\left(N S P_{i}+N S N_{i}\right) \text {. }
$$

Step 8. According to the order from the value of AS, the alternative with the larger value is better. 


\section{Numerical example and comparative analysis}

\subsection{Numerical example}

The construction enterprise to be in an invincible position in the new round of competition in the market, it must adapt to the changing market competition environment, to cultivate their own core competitiveness, the core competitiveness is the low cost strategy. When analyzing the cost control of the construction project, it is found that there are many problems in the current cost control, especially the cost control methods are backward and rough, and the empirical elements are too many, and there is no credible basis. If you can't carry out effective cost control, the prospects are imaginable. Therefore, it is very important to develop and improve the cost control methods of construction projects to enhance the core competitiveness of enterprises. Obviously, selecting best construction projects is a classical MAGDM issues (Gao et al., 2020). In such section, we offer a computational example to choose a best construction project through using P2TLN-EDAS method. There are five construction projects $P_{i}(i=1,2,3,4,5)$ to be assessed under these beneficial attributes $\alpha_{j}(j=1,2,3,4)$ : (1) $\alpha_{1}$ is the human factors; (2) $\alpha_{2}$ is the security factors; (3) $\alpha_{3}$ is the management factors; (4) $\alpha_{4}$ is the environmental factors. Three experts $D^{k}$ (expert's weight $\zeta=(0.29,0.34,0.37)$ use P2TLNs to assess five construction projects $P_{i}(i=1,2,3,4,5)$ under four attributes (attributes' weight $\omega=(0.15,0.33,0.21,0.31))$.

The following computational steps are included to choose the most desirable construction project.

Step 1. Build three evaluation matrix which could be depicted in Tables 1-3.

Table 1. The evaluation result by $\mathrm{DM}_{1}$ with P2TLNs

\begin{tabular}{|c|c|c|c|c|}
\hline & $\alpha_{1}$ & $\alpha_{2}$ & $\alpha_{3}$ & $\alpha_{4}$ \\
\hline $\mathrm{P}_{1}$ & $<\left(s_{4}, 0\right),(4 / 10,5 / 10)>$ & $<\left(s_{3}, 0\right),(4 / 10,5 / 10)>$ & $<\left(s_{3}, 0\right),(2 / 10,7 / 10)>$ & $<\left(s_{1}, 0\right),(3 / 10,2 / 10)>$ \\
\hline $\mathrm{P}_{2}$ & $<\left(s_{2}, 0\right),(5 / 10,6 / 10)>$ & $<\left(s_{5}, 0\right),(6 / 10,3 / 10)>$ & $<\left(s_{1}, 0\right),(8 / 10,7 / 10)>$ & $<\left(s_{3}, 0\right),(5 / 10,6 / 10)>$ \\
\hline $\mathrm{P}_{3}$ & $<\left(s_{4}, 0\right),(7 / 10,3 / 10)>$ & $<\left(s_{1}, 0\right),(4 / 10,4 / 10)>$ & $<\left(s_{5}, 0\right),(4 / 10,4 / 10)>$ & $<\left(s_{3}, 0\right),(6 / 10,4 / 10)>$ \\
\hline $\mathrm{P}_{4}$ & $<\left(s_{2}, 0\right),(4 / 10,5 / 10)>$ & $<\left(s_{3}, 0\right),(8 / 10,5 / 10)>$ & $<\left(s_{5}, 0\right),(6 / 10,3 / 10)>$ & $<\left(s_{2}, 0\right),(2 / 10,1 / 10)>$ \\
\hline $\mathrm{P}_{5}$ & $<\left(s_{4}, 0\right),(6 / 10,2 / 10)>$ & $<\left(s_{3}, 0\right),(5 / 10,8 / 10)>$ & $<\left(s_{2}, 0\right),(8 / 10,5 / 10)>$ & $<\left(s_{4}, 0\right),(2 / 10,7 / 10)>$ \\
\hline
\end{tabular}

Table 2. The evaluation result by $\mathrm{DM}_{2}$ with P2TLNs

\begin{tabular}{|c|c|c|c|c|}
\hline & $\alpha_{1}$ & $\alpha_{2}$ & $\alpha_{3}$ & $\alpha_{4}$ \\
\hline $\mathrm{P}_{1}$ & $<\left(s_{3}, 0\right),(5 / 10,5 / 10)>$ & $<\left(s_{4}, 0\right),(8 / 10,4 / 10)>$ & $<\left(s_{3}, 0\right),(5 / 10,8 / 10)>$ & $<(s 4,0),(2 / 10,5 / 10)>$ \\
\hline $\mathrm{P}_{2}$ & $<\left(s_{4}, 0\right),(7 / 10,8 / 10)>$ & $<\left(s_{2}, 0\right),(7 / 10,2 / 10)>$ & $<\left(s_{5}, 0\right),(4 / 10,0.9)>$ & $<\left(s_{3}, 0\right),(4 / 10,7 / 10)>$ \\
\hline $\mathrm{P}_{3}$ & $<\left(s_{2}, 0\right),(3 / 10,4 / 10)>$ & $\left\langle\left(s_{1}, 0\right),(5 / 10,8 / 10)>\right.$ & $\left\langle\left(s_{2}, 0\right),(3 / 10,7 / 10)>\right.$ & $<\left(s_{2}, 0\right),(6 / 10,6 / 10)>$ \\
\hline $\mathrm{P}_{4}$ & $<\left(s_{2}, 0\right),(5 / 10,1 / 10)>$ & $<\left(s_{5}, 0\right),(6 / 10,6 / 10)>$ & $<\left(s_{3}, 0\right),(5 / 10,5 / 10)>$ & $<\left(s_{5}, 0\right),(5 / 10,3 / 10)>$ \\
\hline $\mathrm{P}_{5}$ & $<\left(s_{5}, 0\right),(6 / 10,3 / 10)>$ & $<\left(s_{4}, 0\right),(2 / 10,4 / 10)>$ & $<\left(s_{4}, 0\right),(4 / 10,6 / 10)>$ & $<\left(s_{4}, 0\right),(1 / 10,2 / 10)>$ \\
\hline
\end{tabular}


Table 3. The evaluation result by $\mathrm{DM}_{3}$ with P2TLNs

\begin{tabular}{|c|c|c|c|c|}
\hline & $\alpha_{1}$ & $\alpha_{2}$ & $\alpha_{3}$ & $\alpha_{4}$ \\
\hline $\mathrm{P}_{1}$ & $<\left(s_{5}, 0\right),(4 / 10,5 / 10)>$ & $<\left(s_{1}, 0\right),(4 / 10,5 / 10)>$ & $<\left(s_{3}, 0\right),(4 / 10,6 / 10)>$ & $<\left(s_{5}, 0\right),(3 / 10,7 / 10)>$ \\
\hline $\mathrm{P}_{2}$ & $<\left(s_{1}, 0\right),(4 / 10,3 / 10)>$ & $<\left(s_{2}, 0\right),(6 / 10,3 / 10)>$ & $<\left(s_{4}, 0\right),(2 / 10,6 / 10)>$ & $<\left(s_{3}, 0\right),(2 / 10,5 / 10)>$ \\
\hline $\mathrm{P}_{3}$ & $<\left(s_{2}, 0\right),(7 / 10,2 / 10)>$ & $<\left(s_{4}, 0\right),(4 / 10,2 / 10)>$ & $<\left(s_{5}, 0\right),(5 / 10,7 / 10)>$ & $<\left(s_{3}, 0\right),(3 / 10,2 / 10)>$ \\
\hline $\mathrm{P}_{4}$ & $<\left(s_{3}, 0\right),(3 / 10,5 / 10)>$ & $<\left(s_{4}, 0\right),(2 / 10,5 / 10)>$ & $<\left(s_{1}, 0\right),(7 / 10,2 / 10)>$ & $<\left(s_{2}, 0\right),(1 / 10,6 / 10)>$ \\
\hline $\mathrm{P}_{5}$ & $<\left(s_{4}, 0\right),(6 / 10,7 / 10)>$ & $<\left(s_{5}, 0\right),(3 / 10,1 / 10)>$ & $<\left(s_{2}, 0\right),(1 / 10,6 / 10)>$ & $<\left(s_{5}, 0\right),(5 / 10,1 / 10)>$ \\
\hline
\end{tabular}

Step 2. Employ the P2TLWA operator to aggregate these three group matrix (expert's weight $\zeta=(0.29,0.34,0.37)$. The results could be depicted in Table 4 .

Table 4. The fused results by P2TLWA operator

\begin{tabular}{|c|c|c|}
\hline & $\alpha_{1}$ & $\alpha_{2}$ \\
\hline $\mathrm{P}_{1}$ & $<\left(\mathrm{s}_{4}, 0.0300\right),(0.4379,0.6113)>$ & $<\left(\mathrm{s}_{3},-0.4000\right),(0.6085,0.5667)>$ \\
\hline $\mathrm{P}_{2}$ & $<\left(\mathrm{s}_{2}, 0.3100\right),(0.5604,0.5937)>$ & $<\left(\mathrm{s}_{3},-0.1300\right),(0.6384,0.3706)>$ \\
\hline $\mathrm{P}_{3}$ & $<\left(\mathrm{s}_{3},-0.4200\right),(0.6157,0.4037)>$ & $<\left(\mathrm{s}_{2}, 0.1100\right),(0.4379,0.5110)>$ \\
\hline $\mathrm{P}_{4}$ & $<\left(\mathrm{s}_{2}, 0.3700\right),(0.4092,0.3537)>$ & $<\left(\mathrm{s}_{4}, 0.0500\right),(0.6088,0.6504)>$ \\
\hline $\mathrm{P}_{5}$ & $<\left(\mathrm{s}_{4}, 0.3400\right),(0.6000,0.5820)>$ & $<\left(\mathrm{s}_{4}, 0.0800\right),(0.3519,0.3124)>$ \\
\hline & $\alpha_{3}$ & $\alpha_{4}$ \\
\hline $\mathrm{P}_{1}$ & $<\left(\mathrm{s}_{3}, 0.0000\right),(0.3998,0.7673)>$ & $<\left(\mathrm{s}_{4},-0.5000\right),(0.2707,0.6924)>$ \\
\hline $\mathrm{P}_{2}$ & $<\left(\mathrm{s}_{3}, 0.4700\right),(0.5565,0.7987)>$ & $<\left(\mathrm{s}_{3}, 0.0000\right),(0.3821,0.6854)>$ \\
\hline $\mathrm{P}_{3}$ & $<\left(\mathrm{s}_{4},-0.0200\right),(0.4151,0.7763)>$ & $<\left(\mathrm{s}_{3},-0.3400\right),(0.5206,0.4634)>$ \\
\hline $\mathrm{P}_{4}$ & $<\left(\mathrm{s}_{3},-0.1600\right),(0.6156,0.4355)>$ & $<\left(\mathrm{s}_{3}, 0.0200\right),(0.3274,0.5497)>$ \\
\hline $\mathrm{P}_{5}$ & $<\left(\mathrm{s}_{3},-0.3200\right),(0.5494,0.6958)>$ & $<\left(\mathrm{s}_{4}, 0.3700\right),(0.3385,0.2468)>$ \\
\hline
\end{tabular}

Step 4. Calculate the average solution (AV) by formula (13):

$$
\begin{aligned}
&\left.A V_{1}=\left\{\begin{array}{l}
\Delta\left(\begin{array}{l}
\left.\frac{1}{5} \times 4.03+\frac{1}{5} \times 2.31+\frac{1}{5} \times 2.58+\frac{1}{5} \times 2.37+\frac{1}{5} \times 4.34\right) \\
\left(\begin{array}{l}
1-\left(1-0.4379^{2}\right)^{\frac{1}{5}} \times\left(1-0.5604^{2}\right)^{\frac{1}{5}} \times\left(1-0.6157^{2}\right)^{\frac{1}{5}} \\
\times\left(1-0.4092^{2}\right)^{\frac{1}{5}} \times\left(1-0.6000^{2}\right)^{\frac{1}{5}}
\end{array}\right. \\
(0.6113)^{\frac{1}{5}} \times(0.5937)^{\frac{1}{5}} \times(0.4037)^{\frac{1}{5}} \times(0.3537)^{\frac{1}{5}} \times(0.5820)^{\frac{1}{5}}
\end{array}\right.
\end{array}\right)\right\} \\
&\left\langle\left(\mathrm{s}_{3}, 0.1260\right),(0.5363,0.4965)\right\rangle ;
\end{aligned}
$$




$$
\left.\begin{array}{rl}
A V_{2}=\left\{\begin{array}{l}
\Delta\left(\frac{1}{5} \times 2.6+\frac{1}{5} \times 2.87+\frac{1}{5} \times 2.11+\frac{1}{5} \times 4.05+\frac{1}{5} \times 4.08\right), \\
1-\left(1-0.6085^{2}\right)^{\frac{1}{5}} \times\left(1-0.6384^{2}\right)^{\frac{1}{5}} \times\left(1-0.4379^{2}\right)^{\frac{1}{5}} \\
\times\left(1-0.6088^{2}\right)^{\frac{1}{5}} \times\left(1-0.3519^{2}\right)^{\frac{1}{5}}
\end{array}\right.
\end{array}\right\}=
$$

So the values of $A V_{j}$ are given as follows.

$$
A V_{1 \times 4}=\left\{\begin{array}{l}
\left\langle\left(s_{3}, 0.1260\right),(0.5363,0.4965)\right\rangle,\left\langle\left(s_{3}, 0.1420\right),(0.5491,0.4653)\right\rangle, \\
\left.\left(s_{3}, 0.1940\right),(0.5191,0.6788)\right\rangle,\left\langle\left(s_{3}, 0.3100\right),(0.3812,0.4954)\right\rangle
\end{array}\right\} .
$$

Step 5. Through the results of AV, determine the PDA and NDA by equation (14) and (15) (See Tables 5-7).

Step 6. Compute $S P_{i}$ and $S N_{i}$ by equation (18) (weighting vector $\omega=(0.15,0.33,0.21,0.31)$, the results are depicted as:

$$
\begin{aligned}
& S P_{1}=0.0019, S P_{2}=0.0229, S P_{3}=0.0000, S P_{4}=0.1061, S P_{5}=0.2988 \\
& S N_{1}=0.2303, S N_{2}=0.1669, S N_{3}=0.1886, S N_{4}=0.0931, S N_{5}=0.0318
\end{aligned}
$$


Table 5. The scores of $g_{i j}$ and $A V_{j}$

\begin{tabular}{|c|c|c|c|c|}
\hline & $\alpha_{1}$ & $\alpha_{2}$ & $\alpha_{3}$ & $\alpha_{4}$ \\
\hline $\mathrm{P}_{1}$ & 1.6483 & 1.3639 & 0.8566 & 1.0394 \\
\hline $\mathrm{P}_{2}$ & 1.1105 & 1.8228 & 1.1657 & 1.0143 \\
\hline $\mathrm{P}_{3}$ & 1.5687 & 0.9818 & 1.1336 & 1.4048 \\
\hline $\mathrm{P}_{4}$ & 1.2352 & 1.9190 & 1.6888 & 1.2155 \\
\hline $\mathrm{P}_{5}$ & 2.2162 & 2.0935 & 1.0957 & 2.3023 \\
\hline$A V_{j}$ & 1.6273 & 1.7046 & 1.2913 & 1.4893 \\
\hline
\end{tabular}

Table 6. The results of $P D A_{i j}$

\begin{tabular}{|c|c|c|c|c|}
\hline & $\alpha_{1}$ & $\alpha_{2}$ & $\alpha_{3}$ & $\alpha_{4}$ \\
\hline$P_{1}$ & 0.0129 & 0 & 0 & 0 \\
\hline$P_{2}$ & 0 & 0.0693 & 0 & 0 \\
\hline$P_{3}$ & 0 & 0 & 0 & 0 \\
\hline$P_{4}$ & 0 & 0.1258 & 0.3078 & 0 \\
\hline$P_{5}$ & 0.3619 & 0.2282 & 0 & 0.5458 \\
\hline
\end{tabular}

Table 7. The results of $N D A_{i j}$

\begin{tabular}{|c|c|c|c|c|}
\hline & $\alpha_{1}$ & $\alpha_{2}$ & $\alpha_{3}$ & $\alpha_{4}$ \\
\hline$P_{1}$ & 0 & 0.1999 & 0.3366 & 0.3021 \\
\hline$P_{2}$ & 0.3176 & 0 & 0.0973 & 0.3190 \\
\hline$P_{3}$ & 0.0360 & 0.4240 & 0.1221 & 0.0568 \\
\hline$P_{4}$ & 0.2410 & 0 & 0 & 0.1838 \\
\hline$P_{5}$ & 0 & 0 & 0.1515 & 0 \\
\hline
\end{tabular}

Step 7. Normalize the values obtained by Step 6 according to formula (19) and the results are recorded as follows:

$$
\begin{aligned}
& N S P_{1}=0.0065, N S P_{2}=0.0766, N S P_{3}=0.0000, N S P_{4}=0.3552, N S P_{5}=1.0000 \\
& N S N_{1}=0.000, N S N_{2}=0.2751, N S N_{3}=0.1812, N S N_{4}=0.5956, N S N_{5}=0.8619
\end{aligned}
$$

Step 8. Acquire AS on the foundation of $N S P_{i}$ and $N S N_{i}$.

$$
A S_{1}=0.0032, A S_{2}=0.1759, A S_{3}=0.0906, A S_{4}=0.4754, A S_{5}=0.9309 \text {. }
$$

Step 9. Obviously, the order of all alternatives is $P_{5}>P_{4}>P_{2}>P_{3}>P_{1}$ and the best of five alternatives is $\mathrm{P}_{5}$.

\subsection{Comparative studies}

\subsubsection{Compare with existing operators}

The P2TLN-EDAS method is compared with P2TLWA and P2TLWG operator (Wei et al., 2017). The final computational values are depicted in the Tables 8-9. 
Table 8. The fusing values of P2TLWA \& P2TLWG operators

\begin{tabular}{|c|c|c|}
\hline & P2TLWA operator & P2TLWG operator \\
\hline $\mathrm{P}_{1}$ & $<\left(\mathrm{s}_{3}, 0.1775\right),(0.4659,0.6500)>$ & $<\left(\mathrm{s}_{3}, 0.1376\right),(0.4126,0.6663)>$ \\
\hline $\mathrm{P}_{2}$ & $<\left(\mathrm{s}_{3},-0.0477\right),(0.5473,0.5655)>$ & $<\left(\mathrm{s}_{3},-0.069\right),(0.5188,0.6386)>$ \\
\hline $\mathrm{P}_{3}$ & $<\left(\mathrm{s}_{3},-0.2563\right),(0.4937,0.5224)>$ & $<\left(\mathrm{s}_{3},-0.3304\right),(0.4808,0.5714)>$ \\
\hline $\mathrm{P}_{4}$ & $<\left(\mathrm{s}_{3}, 0.2246\right),(0.5202,0.5179)>$ & $<\left(\mathrm{s}_{3}, 0.1672\right),(0.4743,0.5481)>$ \\
\hline $\mathrm{P}_{5}$ & $<\left(\mathrm{s}_{4},-0.0851\right),(0.4478,0.3772)>$ & $<\left(\mathrm{s}_{4},-0.1488\right),(0.4136,0.4739)>$ \\
\hline
\end{tabular}

Table 9. Score results of alternatives

\begin{tabular}{|c|c|c|c|c|c|c|}
\hline \multirow{2}{*}{$\begin{array}{c}\text { Method } \\
\text { name }\end{array}$} & & \multicolumn{4}{|c|}{ Scores } & \multirow{2}{*}{ Order } \\
\cline { 2 - 6 } & $\mathrm{P}_{1}$ & $\mathrm{P}_{2}$ & $\mathrm{P}_{3}$ & $\mathrm{P}_{4}$ & $\mathrm{P}_{5}$ & \\
\hline P2TLWA & 1.2625 & 1.4463 & 1.3318 & 1.6160 & 2.0714 & $\mathrm{P}_{5}>\mathrm{P}_{4}>\mathrm{P}_{2}>\mathrm{P}_{3}>\mathrm{P}_{1}$ \\
\hline P2TLWG & 1.1393 & 1.2623 & 1.2075 & 1.4642 & 1.8225 & $\mathrm{P}_{5}>\mathrm{P}_{4}>\mathrm{P}_{2}>\mathrm{P}_{3}>\mathrm{P}_{1}$ \\
\hline
\end{tabular}

\subsubsection{Compare with P2TL-TODIM}

Then, we compare the P2TLN-EDAS method with P2TL-TODIM method (Huang \& Wei, 2018). The computational results are depicted in Table 10.

Table 10. Order of five alternatives

\begin{tabular}{|l|c|}
\hline \multicolumn{1}{|c|}{ Algorithms } & Order \\
\hline P2TLN-EDAS & $\mathrm{P}_{5}>\mathrm{P}_{4}>\mathrm{P}_{2}>\mathrm{P}_{3}>\mathrm{P}_{1}$ \\
\hline P2TLN-TODIM & $\mathrm{P}_{5}>\mathrm{P}_{3}>\mathrm{P}_{4}>\mathrm{P}_{2}>\mathrm{P}_{1}$ \\
\hline
\end{tabular}

It is clear that P2TLN-EDAS method in contrast to P2TLWA/P2TLWG operator and P2TLN-TODIM, the same ranking is obtained. Moreover, P2TLN-EDAS method considers conflicting attributes to improve the accuracy and effectiveness of MAGDM application. In our subsequent works, the proposed P2TLN-EDAS method shall be extended to other uncertain MAGDM (Gao et al., 2019, 2020) and fuzzy MAGDM issues (Kou et al., 2016; Wang et al., 2019b; Wei et al., 2019).

\section{Conclusions}

In a MAGDM method, the uncertainty and hesitancy are always inevitable. The P2TLSs are easier to reflect uncertain or fuzzy information. Meanwhile, in contrast to other MAGDM approaches, such as TOPSIS, VIKOR and TODIM algorithms, EDAS algorithm is more reliable and easier to compute when different weights for attributes are given and it has required fewer computations. Thus, in this article, the P2TLN-EDAS method for MAGDM problems with P2TLNs is developed. Firstly, the fundamental concepts of P2TLNs are introduced as prerequisite knowledge. Then, the P2TLNs of each decision maker (DMs) are fused by using 
the P2TLWA and P2TLWG operators. Next, we describe the steps of EDAS method briefly. Furthermore, we combine EDAS method with P2TLNs information, P2TLN-EDAS that is a new MAGDM method is constructed and the computing steps are simply listed. Finally, we use a computational example and a comparative analysis to verify its effectiveness and practicality. We will carry out further studies in some other uses of this method such as risk analysis and other domains.

\section{Acknowledgements}

This paper is supported by the National Natural Science Foundation of China (No. 71571128) and the Humanities and Social Sciences Foundation of Ministry of Education of the People's Republic of China (16YJA840008).

\section{References}

Chen, T. Y. (2018). A novel PROMETHEE-based outranking approach for multiple criteria decision analysis with pythagorean fuzzy information. IEEE Access, 6, 54495-54506. https://doi.org/10.1109/ACCESS.2018.2869137

Gao, H. (2018). Pythagorean fuzzy Hamacher Prioritized aggregation operators in multiple attribute decision making. Journal of Intelligent \& Fuzzy Systems, 35, 2229-2245. https://doi.org/10.3233/JIFS-172262

Gao, H., Lu, M., \& Wei, Y. (2019). Dual hesitant bipolar fuzzy hamacher aggregation operators and their applications to multiple attribute decision making. Journal of Intelligent \& Fuzzy Systems, 37, 5755-5766. https://doi.org/10.3233/JIFS-18266

Gao, H., Ran, L. G., Wei, G. W., Wei, C., \& Wu, J. (2020). VIKOR method for MAGDM based on q-rung interval-valued orthopair fuzzy information and its application to supplier selection of medical consumption products. International Journal of Environmental Research and Public Health, 17, 525. https://doi.org/10.3390/ijerph17020525

He, T. T., Wei, G. W., Lu, J. P., Wei, C., \& Lin, R. (2019). Pythagorean 2-tuple linguistic Taxonomy method for supplier selection in medical instrument industries. International Journal of Environmental Research and Public Health, 16, 4875. https://doi.org/10.3390/ijerph16234875

He, Y., Lei, F., Wei, G. W., Wang, R., Wu, J., \& Wei, C. (2019). EDAS method for multiple attribute group decision making with probabilistic uncertain linguistic information and its application to green supplier selection. International Journal of Computational Intelligence Systems, 12, 1361-1370. https://doi.org/10.2991/ijcis.d.191028.001

Herrera, F., \& Martinez, L. (2001). The 2-tuple linguistic computational model. Advantages of its linguistic description, accuracy and consistency. International Journal of Uncertainty Fuzziness and Knowledge-Based Systems, 9, 33-48. https://doi.org/10.1142/S0218488501000971

Huang, Y. H., \& Wei, G. W. (2018). TODIM method for Pythagorean 2-tuple linguistic multiple attribute decision making. Journal of Intelligent \& Fuzzy Systems, 35, 901-915.

https://doi.org/10.3233/JIFS-171636

Ilieva, G. (2018). Group decision analysis algorithms with EDAS for interval fuzzy sets. Cybernetics and Information Technologies, 18, 51-64. https://doi.org/10.2478/cait-2018-0027

Keshavarz Ghorabaee, M., Amiri, M., Zavadskas, E. K., Turskis, Z., \& Antucheviciene, J. (2017a). A new multi-criteria model based on interval type-2 fuzzy sets and EDAS method for supplier evaluation 
and order allocation with environmental considerations. Computers \& Industrial Engineering, 112, 156-174. https://doi.org/10.1016/j.cie.2017.08.017

Keshavarz Ghorabaee, M., Amiri, M., Zavadskas, E. K., Turskis, Z., \& Antucheviciene, J. (2017b). Stochastic EDAS method for multi-criteria decision-making with normally distributed data. Journal of Intelligent \& Fuzzy Systems, 33, 1627-1638. https://doi.org/10.3233/JIFS-17184

Keshavarz Ghorabaee, M., Zavadskas, E. K., Amiri, M., \& Turskis, Z. (2016). Extended EDAS method for fuzzy multi-criteria decision-making: An application to supplier selection. International Journal of Computers Communications \& Control, 11, 358-371. https://doi.org/10.15837/ijccc.2016.3.2557

Keshavarz Ghorabaee, M., Zavadskas, E. K., Olfat, L., \& Turskis, Z. (2015). Multi-criteria inventory classification using a new method of evaluation based on distance from average solution (EDAS). Informatica, 26, 435-451. https://doi.org/10.15388/Informatica.2015.57

Kou, G., Ergu, D., Lin, C. S., \& Chen, Y. (2016). Pairwise comparison matrix in multiple criteria decision making. Technological and Economic Development of Economy, 22, 738-765. https://doi.org/10.3846/20294913.2016.1210694

Lei, F., Wei, G. W., Lu, J. P., Wei, C., \& Wu, J. (2019). GRA method for probabilistic linguistic multiple attribute group decision making with incomplete weight information and its application to waste incineration plants location problem. International Journal of Computational Intelligence Systems, 12, 1547-1556. https://doi.org/10.2991/ijcis.d.191203.002

Li, Z. X., \& Lu, M. (2019). Some novel similarity and distance and measures of Pythagorean fuzzy sets and their applications. Journal of Intelligent \& Fuzzy Systems, 37, 1781-1799. https://doi.org/10.3233/JIFS-179241

Li, Z. X., Wei, G. W., Wang, R., Wu, J., Wei, C., \& Wei, Y. (2020). EDAS method for multiple attribute group decision making under q-rung orthopair fuzzy environment. Technological and Economic Development of Economy, 26, 86-102. https://doi.org/10.3846/tede.2019.11333

Lu, J. P., \& Wei, C. (2019). TODIM method for performance appraisal on social-integration-based rural reconstruction with interval-valued intuitionistic fuzzy information. Journal of Intelligent \& Fuzzy Systems, 37, 1731-1740. https://doi.org/10.3233/JIFS-179236

Morente-Molinera, J. A., Kou, G., Peng, Y., Torres-Albero, C., \& Herrera-Viedma, E. (2018). Analysing discussions in social networks using group decision making methods and sentiment analysis. Information Sciences, 447, 157-168. https://doi.org/10.1016/j.ins.2018.03.020

Peng, X. D., \& Yang, Y. (2015). Some results for pythagorean fuzzy sets. International Journal of Intelligent Systems, 30, 1133-1160. https://doi.org/10.1002/int.21738

Ren, P. J., Xu, Z. S., \& Gou, X. J. (2016). Pythagorean fuzzy TODIM approach to multi-criteria decision making. Applied Soft Computing, 42, 246-259. https://doi.org/10.1016/j.asoc.2015.12.020

Stevic, Z., Vasiljevic, M., Zavadskas, E. K., Sremac, S., \& Turskis, Z. (2018). Selection of carpenter manufacturer using fuzzy EDAS method. Inzinerine Ekonomika-Engineering Economics, 29, 281-290. https://doi.org/10.5755/j01.ee.29.3.16818

Wang, J., Gao, H., \& Lu, M. (2019a). Approaches to strategic supplier selection under interval neutrosophic environment. Journal of Intelligent \& Fuzzy Systems, 37, 1707-1730. https://doi.org/10.3233/JIFS-179235

Wang, J., Lu, J. P., Wei, G. W., Lin, R., \& Wei, C. (2019b). Models for MADM with Single-valued neutrosophic 2-tuple linguistic muirhead mean operators. Mathematics, 7, 442. https://doi.org/10.3390/math7050442

Wang, R. (2019). Research on the application of the financial investment risk appraisal models with some interval number muirhead mean operators. Journal of Intelligent \& Fuzzy Systems, 37, 17411752. https://doi.org/10.3233/JIFS-179237 
Wei, G. W., Lu, M., Alsaadi, F. E., Hayat, T., \& Alsaedi, A. (2017). Pythagorean 2-tuple linguistic aggregation operators in multiple attribute decision making. Journal of Intelligent \& Fuzzy Systems, 33, 1129-1142. https://doi.org/10.3233/JIFS-16715

Wei, G. W., Wei, C., Wu, J., \& Wang, H. J. (2019). Supplier selection of medical consumption products with a probabilistic linguistic MABAC method. International Journal of Environmental Research and Public Health, 16, 5082. https://doi.org/10.3390/ijerph16245082

Wei, G. W., Zhang, S. Q., Lu, J. P., Wu, J., \& Wei, C. (2019). An extended bidirectional projection method for picture fuzzy MAGDM and its application to safety assessment of construction project. IEEE Access, 7, 166138-166147. https://doi.org/10.1109/ACCESS.2019.2953316

Wu, L. P., Gao, H., \& Wei, C. (2019a). VIKOR method for financing risk assessment of rural tourism projects under interval-valued intuitionistic fuzzy environment. Journal of Intelligent \& Fuzzy Systems, 37, 2001-2008. https://doi.org/10.3233/JIFS-179262

Wu, L. P., Wang, J., \& Gao, H. (2019b). Models for competiveness evaluation of tourist destination with some interval-valued intuitionistic fuzzy Hamy mean operators. Journal of Intelligent \& Fuzzy Systems, 36, 5693-5709. https://doi.org/10.3233/JIFS-181545

Yager, R. R. (2014). Pythagorean membership grades in multicriteria decision making. IEEE Transactions on Fuzzy Systems, 22, 958-965. https://doi.org/10.1109/TFUZZ.2013.2278989 